\section{Hautkrebsrisiko steigt mit der Anzahl der UVB-Behandlungen}

\author{
Die Breit- und Schmalband-UVB-Fototherapie ist bei einer \\ generalisierten Psoriasis die Methode der Wahl, wenn die \\ Erkrankung weit fortgeschritten ist. Obwohl bekannt ist, \\ dass die UVB-Strahlung mit einem erhöhten Hautkrebsrisiko \\ einhergeht, gilt die UVB-Therapie als sicher. \\ Acta Derm Venereol 2014; 94: 425-430
}

Die Wissenschaftler um A. Osmancevic haben in ihrer Studie die kumulative Hautkrebsrate bei Psoriasis-Patienten, die sich einer UVB-Fototherapie unterzogen hatten, ermittelt. Zwischen 2006 und 2009 nahmen 162 hellhäutige PsoriasisPatienten aus Westschweden an der Querschnittsstudie teil. 116 der 162 Patienten waren männlich und 46 weiblich. Das Durchschnittsalter betrug 56,0 $\pm 13,5$ Jahre. Alle Studienteilnehmer wurden in den vorangegangenen 5 Jahren mehr als 100mal mithilfe von UVB-Strahlung behandelt. Die Autoren bestimmten das Haut- krebsrisiko und untersuchten, ob die Art des Leuchtmittels (Breitband- oder Schmalband-UVB-Lampen) bzw. die Anzahl der Behandlungen das Risiko beeinflussen.

\section{Die Art des Leuchtmittels beeinflusst das Krebsrisiko nicht $\nabla$}

8 Patienten (4,9\%) hatten histopathologisch bestätigten Hautkrebs. Im Jahr 2008 betrug die kumulative Hautkrebsrate bei Schweden im Alter zwischen 60 und 69
Jahren 9,6\%. Innerhalb der Studie lag die geschätzte kumulative Hautkrebsrate unter den Psoriasis-Patienten derselben Altersgruppe bei $8,2 \%$, was sich nicht von der Normalbevölkerung unterschied. Die Autoren identifizierten einen positiven Zusammenhang zwischen der Anzahl der UVB-Behandlungen und dem Risiko, an Hautkrebs zu erkranken ( $p=0,012)$. Auch das Alter der Patienten korrelierte positiv mit diesem Risiko $(p=0,049)$. Ob eine Breitband- oder Schmalband-UVB-Fototherapie zum Einsatz kam, wirkte sich hingegen nicht auf das Hautkrebsrisiko aus $(\mathrm{p}=0,31)$.

\section{Fazit}

Die Studienergebnisse zeigen, dass bei Psoriasis-Patienten, die sich einer UVBFototherapie unterziehen, das Hautkrebsrisiko mit der Anzahl der Behandlungen sowie mit zunehmendem Lebensalter ansteigt. Die Art des verwendeten Leuchtmittels habe laut den Autoren hingegen keinen Einfluss. Insgesamt betrachtet lag die geschätzte kumulative Hautkrebsrate unter Psoriasis-Patienten auf dem Niveau der schwedischen Allgemeinbevölkerung.

Dr. Frank Lichert, Weilburg 